\title{
Los sectores agroindustriales de Argentina y Brasil, 1990-2010, y su evolución comparada
}

\section{The agro-industrial sectors of Argentina and Brazil 1990-2010 and compared evolution}

\author{
Mercedes Muro de Nadal \\ Licenciada en Historia \\ murodenadal@gmail.com \\ Susana Merlo \\ Ingeniera Agrónoma
}

Centro de Estudios Agrarios y Agroindustriales (CIEA), Facultad de Ciencias Económicas, Universidad de Buenos Aires (UBA)

Fecha de recepción 03 de febrero de 2014

Fecha de aprobación 15 de julio de 2014

\section{Resumen}

Las economías de Argentina y Brasil marcaron una fuerte impronta productiva y exportadora agroindustrial en las dos últimas décadas. Ambos fueron socios iniciales y promotores de la integración regional en el Mercosur, pero presentaron a lo largo del período una evolución dispar. Los principales factores que marcaron esas diferencias debemos buscarlos en el comportamiento de los mercados internacionales, las innovaciones tecnológicas, el clima, los capitales invertidos y, en especial, la incidencia de las políticas internas de cada país. Una desproporción en el tamaño de sus mercados internos, crisis internacionales, crisis dentro de los países, devaluaciones, relaciones con otros bloques y políticas de diferente signo, fueron algunos de los desafíos por los que transitaron ambos. 
108.

El objetivo de este trabajo es analizar entonces la evolución del sector de la producción agroindustrial en ambos países por medio del análisis comparativo y plantear el debate académico necesario para identificar las causas de las evoluciones dispares; así mismo, sugerir cuáles son las herramientas más útiles para el desarrollo a largo plazo de Argentina o de otros países enfrentados a disyuntivas similares.

Palabras clave: Agroindustria, agricultura, planificación, políticas de desarrollo, recursos naturales y energía, desarrollo económico, cambio tecnológico, investigación y desarrollo.

Clasificación JEL: Q00, Q1, Q2, Q3, Q4 , 01, 03

\begin{abstract}
The economies of Argentina and Brazil, both initial partners and promoters of Mercosur's regional integration program, and both having a strong agro-industrial production and export base, have shown in recent years a disparate evolution. To find the main causes of this disparity we must look at the incidence of internal policies, the behavior of the international markets, differing technological innovations, climate and invested capital.

Different geographic proportions, internal and international crises, devaluations, the implementation of different political models and relationships with different economic blocks, were only some of the challenges faced by both nations.

Aiming to find the steps to be followed by Argentina for its long term development, the objective of this study is to open the academic debate that is necessary to identify the causes of the disparity of economic development through the comparative analysis of the agro industrial production of each country.
\end{abstract}

Keywords: Agribusiness, agriculture, natural resources and y energy, economic development, planning and development policies and technological change, research and development.

JEL codes: Q00, Q1 Q2, Q3, Q4, 01, 02, 03. 


\section{Introducción}

Las economías de Argentina y Brasil fueron socios iniciales y promotores de la integración regional en el Mercosur. Los dos orientaron sus políticas a favorecer la producción y exportación agroindustrial, pero presentaron a lo largo de los últimos años una evolución dispar. Los principales factores que marcaron esas diferencias se encuentran en el comportamiento de los mercados internacionales, las innovaciones tecnológicas, el clima, los capitales invertidos y la incidencia de las políticas internas de cada país.

El análisis de la estructura productiva de cada uno permite hallar las causas del proyecto de integración regional y su evolución hasta nuestros días. El desarrollo estuvo y está condicionado por una serie de variables en las que hay que detenerse: la coordinación macroeconómica alcanzada, la estructura arancelaria, la dinámica de los flujos comerciales intra y extrazona, y el volumen y el tipo de producción agroindustrial, entre otros.

Una desproporción evidente en el tamaño de ambos, crisis internacionales y dentro de los países, devaluaciones, relaciones con otros bloques y políticas de diferente signo, fueron algunos de los desafíos por los que transitaron. En el ámbito de las políticas internas, y a lo largo de los años transcurridos, Argentina y Brasil experimentaron cambios diversos, en ocasiones muy significativos, que generaron coincidencias y desavenencias, avances y retrocesos. La comunidad empresarial, cámaras, asociaciones y gremios, fueron todos actores influyentes en un sentido o en otro, promoviendo políticas públicas que acercaron o alejaron los objetivos propuestos.

Al encarar la identificación - por medio del análisis comparativo, y con eje en el sector de la producción agroindustrial- de las causas de las evoluciones dispares, se busca encontrar las herramientas más útiles para el desarrollo a largo plazo de Argentina, que logren incrementar una producción agropecuaria diversificada, revirtiendo el fuerte retroceso en la producción de rubros tradicionales como la carne vacuna y el trigo, recuperando las saludables rotaciones entre cultivos y otras actividades, y evitando las fuertes transferencias, la expulsión de la mano de obra y los desfases comerciales y medioambientales que impiden el equilibrio del sistema.

En el primer bloque del trabajo se exponen una síntesis de los orígenes del Mercosur, las razones de cada país para participar en el proyecto de integración y la evolución general del bloque en los casi veinte años transcurridos desde que se inició el proceso. En el segundo bloque se analizan el comportamiento del sector agroindustrial en cada país en el período considerado y las características que presenta cada uno en cuanto a evolución de la producción agropecuaria, principales productos, capitales invertidos en agroindustrias, porcentaje agro dentro del Producto Bruto Interno (PBI) de cada país, etcétera. Solo se toman los principales rubros productivos de cada uno que sean comparables entre sí. En el tercer bloque se confrontan los resultados de la evolución de cada uno transcurrido el lapso considerado.

\section{El Mercosur: origen y evolución}

Los años noventa estuvieron marcados en el ámbito regional por la formación del Mercosur, y el acercamiento bilateral iniciado entre Argentina y Brasil a mediados de los años ochenta 
tuvo continuidad en la década siguiente con la firma del Tratado de Asunción, en marzo de 1991, por el cual Argentina, Brasil, Paraguay y Uruguay constituyeron el Mercado Común del Sur o Mercosur. Posteriormente, Bolivia y Chile se sumaron como países asociados. En este sentido, el Tratado fue visto como una oportunidad para atraer inversiones, importar bienes de capital para la modernización de sus empresas y exportar sus productos.

A partir de 1995, con la aplicación del Protocolo de Ouro Preto y su mandato de conformación de la propuesta de Mercado Común, se dio inicio a las tres etapas que comenzarían por una Zona de Libre Comercio, y se hicieron visibles algunos problemas que acompañarían al Mercosur durante su vigencia.

Uno de los primeros signos de conflicto interno se manifestó en el momento de fijar el Arancel Externo Común (AEC) requerido para la conformación de una Unión Aduanera. Las diferencias de las estructuras productivas y de protección de los cuatro países obstaculizaron la fijación de aranceles por producto. Mientras Argentina, Uruguay y Paraguay buscaban menor protección para productos manufacturados y relativamente mayor para el sector agrícola, Brasil proponía medidas diametralmente opuestas. La solución llegó después de arduas negociaciones, en las que igualmente debieron contemplarse innumerables excepciones al AEC. Otro problema que surgió fue el de las Reglas de Origen.

Debido a los problemas generados en la práctica del modelo de integración, se buscó, a través de una nueva agenda, apuntalar la consolidación y profundización del bloque. En el plano de consolidación se trabajó sobre el Código Aduanero del Mercosur, las medidas no arancelarias, los subsidios y el Régimen de Adecuación interno. A su vez, se intentó consolidar los dos sectores que más problemas generaban entre los miembros, como el automotor y el azucarero.

La evolución del Mercosur distingue tres etapas cronológicas de características disímiles: la primera, entre 1991 y 1998, durante la que los Estados parte atravesaron una coyuntura económica favorable, con un rápido crecimiento de los flujos comerciales intrabloque a partir de la desgravación arancelaria, dando la impresión de estar viviendo años dorados en la integración; la segunda, desde 1999 a 2002, caracterizada por volatilidades extremas de las economías, fuertes crisis coyunturales, importante caída de los niveles de actividad y comercio, y ahondamientos de las asimetrías subyacentes; y finalmente, la tercera etapa, que se extiende hasta la actualidad, en la cual los países atraviesan un nuevo período de crecimiento económico y comercial, aunque matizado por los insistentes reclamos por parte de los países más perjudicados y el surgimiento de conflictos sectoriales intrabloque.

Durante el primer período, las economías del Mercosur crecieron con fuerza, apoyadas centralmente por el favorable contexto externo y la recepción de flujos internacionales de capital. Este crecimiento fue acompañado de una expansión aún más pronunciada del intercambio dentro del bloque, que fue la consecuencia lógica de la eliminación de los aranceles.

Luego de la crisis de los mercados emergentes, el impacto sobre la región fue muy profundo y originó el comienzo de una etapa de fuertes volatilidades de los ciclos económicos y una importante caída del comercio intrazona.

El Mercosur tuvo diferentes objetivos para los países iniciadores. Entre los funcionarios argentinos de esa etapa, las opiniones coinciden en afirmar que fue concebido como una 
herramienta de consolidación de la democracia y generación de la transformación productiva de sus integrantes, según Félix Peña, y que el proyecto de integración se alineaba con el cambio en el poder mundial a partir de la caída del Muro de Berlín, cuando el nuevo eje abandonó lo ideológico-militar y pasó a convertirse en económico-tecnológico, según Alietto Guadagni. Lo cierto es que Argentina y Brasil tomaron la decisión de dar un viraje en su manera de insertarse en la economía mundial, inaugurando un cambio geopolítico muy importante y novedoso para ambos países.

La integración regional aportaba no solo un gran mercado de consumidores para Argentina, y el pie indispensable para el liderazgo regional de Brasil, sino un importante punto de apoyo y desplazamiento para la primera (Guadagni, 1992), que a su vez se pensaba convertir a largo plazo en un abastecedor prioritario de productos agroalimenticios de un mercado cautivo. Esta realidad se fue alejando con los años y los avances productivos de sus socios.

Pero lo que marcó un punto de inflexión al inicio fue la decisión política de los presidentes de dar una fuerte impronta al sector empresarial para que empezara a caminar. Otro sector involucrado desde el inicio fue el sociocultural. Con razón, se analizaba que un proyecto de integración debe transitar por el conocimiento mutuo de las culturas en áreas como el idioma, el intercambio artístico y los espectáculos. En este sentido, Brasil llevó la voz cantante con una visión geopolítica regional muy fuerte, basada en los años en los que se constituyó como imperio, marcando con esa concepción su evolución posterior.

El estudio detallado de cada país muestra que Brasil, a comienzos de los años noventa, ya tenía en claro que su principal socio para lograr su ansiado espacio entre los países industrializados era Argentina. La necesidad de asegurarse la provisión de alimentos a largo plazo, una reserva energética adecuada y mano de obra calificada fue más fuertes que su tradicional proteccionismo y le permitieron encarar un completo cambio en su política exterior. Sin embargo, no era tan sencillo llevarlo a la práctica:

[...] a medida que progresa la integración, los intereses económicos se suman, enfrentan, cruzan y entrecruzan tanto que las consecuencias de esa interacción exceden el terreno económico para ingresar abiertamente en lo político. (Cisneros y Piñeiro Iñíguez, 1994, pp. 25 y 26)

Dado que el mayor interés político se encontraba en Brasil, este fue el que impulsó firmemente la obtención de personería jurídica internacional, para que el Mercosur participara como tal en negociaciones internacionales y para que le sirviera de globo de ensayo de manejo de las nuevas reglas globales.

Uruguay estaba geográficamente enclavado en el centro del eje Buenos Aires-São Paulo $y$, tanto por su historia como por sus antecedentes, se veía como la posible plaza financiera del Mercosur. Se sabe que, en general, los países chicos encuentran más ventajas que inconvenientes en un proyecto de integración. Así, aceptó el desafío y aceleró los tiempos para dar cumplimiento a las exigencias del proyecto.

Paraguay, por su parte, se encontraba políticamente atrasado y, por su ubicación mediterránea, estaba casi obligado a buscar una salida al mar. El hecho de unirse a dos países de 
mayor dimensión y crecimiento le significaba la posibilidad de encarar un ordenamiento territorial, político y administrativo que necesitaba urgentemente. El proyecto tardó en tomar forma porque debían regularizarse muchas situaciones internas. Sin embargo, fue finalmente Paraguay el detonante de la consolidación política del proyecto, cuando tuvo lugar el intento de golpe de Estado del general Oviedo, que provocó la incorporación al texto del Tratado de la estratégica Cláusula de Paz. ${ }^{1}$

Es cierto que Argentina, durante el gobierno del Dr. Raúl Alfonsín, ya había dado los pasos iniciales, pero solo pudo concretar los acuerdos de la industria automotriz. En aquel momento se intentaba revertir el aislamiento político internacional y el estancamiento económico generados por la dictadura militar. Apareció entonces la necesidad de articular alianzas regionales que dieran lugar a proyectos económicos comunes, algo que la vuelta de la democracia facilitó considerablemente.

En el siguiente período presidencial, el Dr. Carlos Menem, entusiasmado con la posibilidad de liderar la región, tomó la idea y la enmarcó en el principio geopolítico del presidente Juan Domingo Perón, con el Tratado Argentina, Brasil, Chile (ABC), dándole el impulso político necesario. En julio de 1990, con la firma del Tratado de Buenos Aires, se aceleró el cronograma de integración fijando la fecha 31 de diciembre de 1994 para que comenzara a funcionar el Mercado Común del Sur.

Entre los factores económicos que influyeron en Argentina, se deben mencionar el endeudamiento externo, la asignación de recursos, la investigación y el desarrollo, las restricciones a la transferencia tecnológica, el diseño del sistema económico-comercial financiero global, y mejoras en la productividad y competencia. Existían entonces razones de orden político y geopolítico, razones tecnológicas, de productividad, de costos y precios, de calidad y actualidad de los productos, de capacidad de negociación internacional y, sobre todo, de dinámica de las producciones nacionales que jugaban a favor de los procesos de asociaciones de preferencia. Pero, en síntesis, el hecho es que el Mercosur surgió como una construcción del poder político dado en cada uno de sus integrantes, que se superpuso a las realidades económicas preexistentes.

Con doscientos millones de consumidores, un producto superior a los quinientos millones de dólares y el resultado de las políticas de apertura y transformación en curso por entonces, era lógico suponer que la apuesta iba a ser exitosa.

Luego, con el Cronograma de Las Leñas, en junio de 1992, se logró la concreción de un Plan de Acción realista para encarar el camino crítico durante la transición. Desde allí se envió una clara señal al mercado mundial: el Mercosur era factible y los plazos se iban a cumplir. Se previó, incluso, la participación de los sectores privado, sindical y consumidor. Los objetivos fijados con fecha cierta de cumplimiento establecían -y se cumplieron- que al comenzar 1995, el mercado común habría comenzado a funcionar; también la unión aduanera, con el establecimiento de una tarifa externa común relativamente baja, un cierto grado de coordinación de políticas macroeconómicas y la armonización de legislaciones. Sin embargo, faltaban todavía muchas medidas para el pleno funcionamiento del Mercosur, y más para llegar a las etapas de

1 En 1996, en San Luis, el Mercosur estableció la "cláusula democrática", que obligaba a los Estados y fue vital para la subsistencia de la frágil democracia paraguaya. 
la unión económica y monetaria a la que se aspiraba, pero ya había una serie de decisiones y señales al mundo importantes, aunque en 2010 aún quedaban cien excepciones para Paraguay y Uruguay, y ochenta para Argentina y Brasil.

El estrechamiento de relaciones políticas y económicas con otros países llevó a buscar espacios de ampliación entre países de la región. Así, se formalizó, en junio de 1996, la firma de un Acuerdo de Complementación Económica (ACE) (integración parcial) con Chile; y de otro ACE con Bolivia, en diciembre de ese mismo año, y con Perú en 2003. Colombia, Ecuador y Venezuela lo hicieron en 2004. En 2006, Venezuela solicitó su adhesión en carácter de miembro pleno, con el apoyo de Brasil, Uruguay y Argentina, pero recién logró desbloquear las acciones en 2012, frenadas hasta ese momento por la oposición del Parlamento paraguayo por la constante violación de las libertades públicas registradas en ese país. Esta circunstancia fue vista por muchos como un salto atrás en el proceso de integración, entre ellos, el expresidente de Uruguay Julio María Sanguinetti, quien manifestó que en esa ocasión se "entró por la ventana" aprovechando la coyuntura, sin que mediara la unanimidad de las aprobaciones legislativas, como exige el Tratado de Asunción. ${ }^{2}$ Otros fueron más lejos aún y objetaron la juridicidad de lo actuado, tanto en el planteo de integración de Venezuela como en la "suspensión transitoria" de Paraguay.

La influencia del Mercosur en la región y en el mundo se modificó radicalmente en los últimos veinticinco años en diferentes sentidos. Por un lado, se abrieron enfoques más cooperativos y de menor competencia y desconfianza mutua entre naciones, a partir de la consolidación de los sistemas democráticos. Otro cambio, gradual pero sostenido, fue el desbalance entre los dos principales socios, en detrimento de Argentina. La gran diferencia de tamaño entre ambos países demostró tener un impacto sobre los incentivos para una mayor integración con Brasil, que resultó difícil de precisar y prever, ya que se presentó como una fuente de oportunidades y de amenazas. Por parte de las primeras, justamente, la mayor dimensión de Brasil atrajo actividades productivas que pudieran tener allí su destino comercial, tanto para sectores con ventajas estáticas como para aquellos que pudieran aprovechar la economía de escala y gama y las ventajas del comercio basado en la especialización intraindustrial. Estaba claro que los productores argentinos mejorarían su posición en el mercado regional y las condiciones de competencia en el mercado internacional, y, además, serían un factor de atracción para la Inversión Extranjera Directa (IED). Pero esa misma diferencia de tamaño de ambas economías sería una amenaza para los sectores que mantenían problemas estructurales de competitividad. Las asimetrías en la especialización de cada país también tuvieron consecuencias que estuvieron siempre presentes en la relación bilateral, con percepciones variables entre los diferentes actores.

El hecho es que las exportaciones de productos primarios de Argentina a Brasil, sobre todo agrícolas de clima templado, representaban, desde fines de los años setenta, el $65 \%$ del total de ese país; mientras que la composición de las exportaciones de Brasil a Argentina consistía básicamente en manufacturas, y llegaban al 72,5\%.

2 J. M. Sanguinetti (25/8/2012), Un Mercosur sin leyes ni principios. La Nación, p. 27. 
Un último factor que condicionó el proyecto de integración fueron las asimetrías regulatorias, de la mano del grado de convergencia/divergencia manifestado en las políticas públicas de cada país que influyeron sobre las visiones dominantes en cada período, en torno a la conveniencia o no de intensificar el vínculo bilateral. Sobre todo, teniendo en cuenta que, aun cuando las políticas macroeconómicas fueron volátiles en ambos países en los últimos veinte años, se registraron claras asimetrías entre ambos en las políticas dirigidas al sector productivo en ese período. Si en el quinquenio 1960/65 el peso relativo de cada país como proveedor y mercado del otro era equivalente, para fines de los años setenta esto se había alterado completamente. Argentina había ido perdiendo su relevancia como mercado de destino y origen del comercio exterior brasileño, mientras que había conservado su promedio de, más o menos, un $10 \%$ de sus exportaciones e importaciones totales con Brasil. La acentuación ininterrumpida de esta tendencia fue condicionando la relación bilateral.

En sentido contrario, a mediados de los años noventa, esa diferencia de tamaño benefició las exportaciones argentinas y ayudó al país a recuperarse de los efectos de la "crisis del tequila", cuando se apreció la moneda brasileña y se produjo una fuerte recuperación de su actividad económica tras la implementación del Plan Real.

Por consiguiente, más allá de las diferencias políticas e ideológicas de las gestiones de ambos países, existían otras institucionales y de recursos que se instalaron en las políticas públicas y generaron, a mediano y largo plazo, un condicionante inercial sobre la visión de los costos y los beneficios resultantes de la integración entre ambos países.

\section{El eje agroindustrial São Paulo-Buenos Aires}

La evidencia empírica demuestra que las estructuras productivas de los países del Mercosur se trastocaron en los últimos veinte años. La conformación del bloque influyó en estos cambios, pero no fue ese el único factor explicativo. En líneas generales, Brasil ganó lugar como base productiva manufacturera en la región, mientras que los países más pequeños, Uruguay y Paraguay, vieron acentuar el peso del sector agropecuario. Pero el sector agropecuario como tal tendió a ganar lugar en las estructuras productivas de los cuatro países.

Dentro del sector agropecuario hubo modificaciones parciales en las que la tecnificación de la producción y la consecuente mejora de la productividad fueron las más destacables, junto con el alza marcada en los precios internacionales de los distintos productos. Brasil tendió al autoabastecimiento de productos agrícolas, que se explica por la propia política de Brasil de fomentar estas actividades y demandar más calidad a los productos de Argentina y Uruguay, como ocurrió con trigo y arroz; así como por el hecho de que en todos los países comenzó a ganar lugar de manera importante la producción de soja, desplazando otros granos y/o actividades.

Además, después del colapso de Argentina de 2001, las tendencias ya iniciadas se acentuaron, creciendo mucho la participación de las importaciones brasileñas en el mercado argentino respecto a años anteriores a la crisis. Simultáneamente, se contrajo la participación de Brasil como mercado de destino para las exportaciones argentinas, y Argentina perdió participación en el mercado brasileño de forma sistemática. Esta caída se explica por la mejora en los precios y el aumento en los volúmenes exportados de productos agrícolas, sobre todo 
oleaginosas, que desviaron las corrientes de comercio hacia otras regiones con una estructura más complementaria del comercio.

El alza de los precios internacionales de los productos agropecuarios, en particular de la soja, indujo un proceso de fuertes inversiones de capital en ambos países destinado a la producción agrícola para expandir las exportaciones extrazona. En este sentido, las IED tuvieron una etapa de incremento sostenido hasta 1998 en Argentina y Brasil. A partir de entonces, las sucesivas crisis macroeconómicas y las indefiniciones en el proceso de integración crearon un ambiente desfavorable para Argentina. Brasil continuó atrayendo capitales, captando entre 1990 y 2000 el 60\% de la IED destinada a la región, incrementándose fuertemente en el período 2001-2004 hasta llegar al 91,7\%. Solo en 2004, las IED al país vecino eran el 89,6\% del total.

La pérdida relativa de relevancia de Argentina respecto de Brasil durante los años ochenta se revirtió en la primera mitad de la década siguiente, debido básicamente a los resultados de la integración. Pero, durante la segunda mitad de los años noventa, la economía argentina se rezagó, y las diferencias se incrementaron. Las causas fueron múltiples, pero ciertamente contribuyeron a este retraso la deuda brasileña, la devaluación de Brasil en 1999, el comienzo de la crisis argentina y un Mercosur que no terminaba de institucionalizarse. En los inicios de la segunda década del siglo XXI, Brasil se encuentra desempeñando un papel muy destacado en el ámbito internacional y aspira a ampliarlo como líder de Sudamérica. Es en este sentido que puede interpretarse su iniciativa para la conformación de la Comunidad Sudamericana de Naciones (UNASUR), dado que sus aspiraciones van más allá del bloque del sur del continente. Pese a ello, la alianza con Argentina en el Mercosur sigue siendo un objetivo estratégico por defender. En la actualidad, y con una economía brasileña que ha tomado una gran distancia de la argentina, el proyecto de integración parece diluirse por falta de interés de los participantes, traducido en incumplimientos constantes de las reglas fijadas. Pareciera haberse perdido el objetivo del "regionalismo abierto".

Sin duda, las industrias alimentarias constituyen la actividad más importante de la mayoría de los países que integran el Mercosur. Argentina era, de hecho, el granero de la región, ingresando históricamente a los mercados internacionales con carnes, trigo y sus derivados, lácteos y oleaginosas, productos todos reconocidos mundialmente, en especial, la carne vacuna.

Brasil, a pesar de su extensión continental, internacionalmente estaba más identificado con los productos tropicales y subtropicales como la caña de azúcar, el café, el algodón, el jugo de naranja, etcétera, aunque también tiene regiones especializadas en carnes y lácteos, y otras en oleaginosas. También, muchos productores brasileños se han expandido en los últimos años adquiriendo tierras y encarando explotaciones agropecuarias, básicamente, en las zonas fronterizas de Uruguay y Paraguay, mientras que los productores argentinos prefirieron Uruguay y, algo menos, Bolivia. En general, no hubo productores de la región que vinieran a producir en Argentina, aunque sí a comprar genética animal, algo de genética vegetal (semillas) y maquinarias.

Hasta las últimas caídas fuertes de la producción, por menor área sembrada y por sequías, Argentina abastecía aproximadamente el $10 \%$ de la demanda mundial de trigo de los tres principales importadores del mundo, Egipto, la Unión Europea y Brasil. Tanto, que el país llegó a aportar el 78\% de las importaciones del vecino y, a su vez, el $46 \%$ de las exportaciones argentinas de trigo tenían como destino Brasil. 
En relación con toda América Latina, al informar la Cepal en 2005 los avances producidos en el sector agrícola en las dos últimas décadas, observaba que, si bien todos los países muestran un dinamismo creciente, sobre todo a partir de 1999, no todos tuvieron los grandes incrementos de productividad, de tecnología y de exportaciones que marcaron al Mercosur. Tomando solo la soja, la expansión fue constante en casi toda la región en los últimos veinte años. De hecho, la Cepal reconocía que Brasil y Argentina, por su peso en los mercados internacionales y las posibilidades de expansión interna, tenían potencialmente más peso que los países de la región que firmaron en años recientes acuerdos con Estados Unidos y la Unión Europea.

La organización del proceso productivo, asociado estrechamente a la prestación de servicios de provisión de insumos, crédito, maquinaria, asistencia técnica de alto nivel, transporte, etcétera, pasó a ser la conducta empresarial necesaria para asegurar el éxito en los mercados, incentivada por el notable incremento de la participación de empresas transnacionales (ETN) y de IED de estas y de las empresas traslatinas. Este crecimiento fue acompañado de demanda de mercados no tradicionales para esos productos, como China. Alto grado de modernización, especialización, eficiencia en la cadena de proveedores y enlace con los servicios fueron los que ubicaron a Brasil y a Argentina en los primeros puestos mundiales. A la par de estos buenos resultados que se fueron consolidando en la primera década del siglo XXI, la pobreza y la indigencia siguieron golpeando al continente, que mereció, para la Cepal, el nombre de "modernizante, pero excluyente". En Argentina, Brasil y Uruguay, la agroindustria muestra la mayor participación en la mano de obra manufacturera total, siendo la industria de alimentos la que más aporta al empleo. Las agroindustrias alimentarias representaban, en 2004, el 10\% del PBI en Argentina y el $27 \%$ de su PBI industrial, y el $18 \%$ del PBI industrial de Brasil. La importancia relativa de estas industrias es inversamente proporcional al grado de industrialización de cada país.

Respecto al intercambio comercial con Brasil, si bien desde 2004 la balanza comercial total fue negativa para Argentina, con un déficit en 2009 de 1.504 millones de dólares, el alto desempeño de nuestro sector agropecuario invirtió ese déficit, arrojando la balanza comercial agropecuaria un saldo positivo de 1.976 millones de dólares. Las exportaciones del sector representaban, en 2010, el 22,16\% del total exportado, con un valor de 2.501 millones de dólares, y constituyen la base que justifica que Brasil siga siendo el principal cliente de Argentina.

\section{Cereales: trigo}

El trigo tuvo gran importancia para Brasil en la constitución del Mercosur, ya que dicho país no contaba entonces con capacidad para producirlo, por su clima, mientras que Argentina no solo podía, sino que lo hacía desde siempre. Por eso quedó explícito que Argentina sería el proveedor de trigo de Brasil y que habría un arancel externo alto que desestimulara las compras extrazona. Pero inmediatamente se dispararon la pregunta y una polémica acerca de dónde se ubicaría entonces la industria molinera, muy desarrollada en el país vecino. Era más eficiente procesar en origen, pero Brasil prefería incorporar valor al trigo en su territorio. La discusión tuvo varias etapas, aunque Argentina terminó exportando harina, a pesar de la fuerte oposición de los molineros brasileños. Con una retención actual de $23 \%$ a 
la exportación de trigo, aunque era más baja cuando se implantó en 2002, los brasileños se quejaban de la ventaja de los molineros argentinos. Cuando se eliminaron las retenciones, en 1991, ambas industrias habían quedado en igualdad de condiciones. Pero al reimplantarse los impuestos a la exportación en Argentina, y al no alcanzarse la coordinación efectiva de las políticas macroeconómicas, la cuestión no llegó a dirimirse nunca, y volvemos a encontrar la discusión en 2007, cuando los molineros brasileños llevaron adelante una campaña ante el gobierno de Lula para impedir la entrada de harina argentina, ante el subsidio al consumo interno de farináceas que esta recibía. Se trataba del consumo interno, pero los competidores internacionales, Brasil entre ellos, lo tomaron como una campaña para incrementar las exportaciones subsidiando al trigo. La defensa corrió por cuenta de la Federación Argentina de la Industrias Molinera (FAIM), que por entonces expresaba su disgusto:

Si dentro del ámbito del Mercosur, con una balanza favorable a Brasil de 3.600 millones de u\$s en 2006, no podemos exportar harina de trigo, ¿qué puede exportar la Argentina? ${ }^{3}$

El trigo argentino representó históricamente entre 90 y 95\% del abastecimiento de este producto en el país vecino y continúa siendo la primera posición de exportación del sector, pese a que se redujo mucho los últimos años, merced a las limitaciones implementadas en Argentina a su exportación, así como por la caída de los volúmenes de producción a causa de esa misma política de restricción a las exportaciones de trigo. Esta situación llevó a que Argentina no pudiera cumplir con las necesidades de abastecimiento del país vecino, obligándolo a comprar en terceros mercados (del hemisferio norte), aun perdiendo la preferencia arancelaria con la que cuenta Argentina como socio del Mercado Común.

\section{Oleaginosas: soja}

La producción de soja en Brasil se concentra en las regiones Centro Oeste y Sur, en los estados de Mato Grosso, Mato Grosso do Sul, Goiás, Paraná y Rio Grande do Sul, los cuales representan el $82 \%$ de la producción de soja de este país. Por su parte, la distribución de la siembra de soja en Argentina se fue ampliando a medida que irrumpieron nuevos materiales genéticos que permitieron incorporar más zonas a la producción, y que se consolidaron los precios de la oleaginosa, que fue así desplazando otros cultivos y actividades. De hecho, buena parte de las tierras mixtas de la Pampa Húmeda, que representan unos 5 o 6 millones de hectáreas, dejaron de ser ganaderas para dedicarse a la agricultura de la soja, mientras que los vacunos fueron desplazados a regiones menos beneficiadas, en general, hacia el norte. También localmente, el maíz sufrió el avance de la soja, más resistente y de cultivo comparativamente más barato, por lo que el cereal perdió entre 1 y 2 millones de hectáreas por el avance de la oleaginosa.

Este crecimiento llevó a que Argentina se ubicara en un destacado lugar: principal productor mundial de harina y aceite de soja, abasteciendo el $44 \%$ de la demanda mundial. A lo largo

3 Polémica en el Mercosur: Industria brasileña insiste con bloquear la importación de harina argentina (07/02/2007). El Informatorio. 
de la crisis de 2001, este cultivo presentó un comportamiento anticíclico que atemperó la caída general de la actividad económica, en medio de un ámbito internacional de precios desfavorables. La fuerte expansión sojera de Argentina y Brasil repercutió, a su vez, en los países vecinos, por medio de fuertes inversiones de empresarios de ambos países en Bolivia, Paraguay y Uruguay (Lapitz, Eviay Gudynas, 2004, p. 64). En Brasil, con el aumento de la importancia de la soja en el mercado internacional, esta pasó a disputar con el maíz áreas de cultivo de verano, llevando a más productores a optar por la oleaginosa, como ocurrió en Argentina.

En la tabla 1, que registra los volúmenes de los productos comparables en el período de Brasil, se puede observar que, en general, el sector agropecuario de Brasil mostró gran desarrollo en el período considerado, aunque la producción de soja estuvo entre las de mayor expansión, con un crecimiento punta a punta de casi 400\%. Además, es la actividad que tiene un mayor peso relativo dentro del total de la producción agrícola de Brasil. En el caso de Argentina, registrado en la tabla 2, se observa que el mayor crecimiento del período se produjo en la producción de soja y maíz, con una disminución del stock ganadero y una notable disminución del área sembrada de trigo.

Tabla 1.

Brasil: producción 1990-2010

\begin{tabular}{|c|c|c|c|}
\hline PRODUCTO & $\mathbf{1 9 9 0 / 1 9 9 1}$ & $\mathbf{2 0 0 0 / 2 0 0 1}$ & $\mathbf{2 0 1 0 / 2 0 1 0}$ \\
\hline Leche (mill. It) & 15.7 & 19.3 & 30.0 \\
\hline Carne vacuna (mill. tn) & & & 9.2 \\
\hline Stock vacuno (mill. cabezas) & 141.7 & 170.2 & 205.3 \\
\hline Maíz producción (mill. tn) & 22 & 34.5 & 55 \\
\hline Máízárea (mill. ha) & 12 & 12 & 13 \\
\hline Trigo producción (mill. tn) & 3.30 & 1.66 & 5.9 \\
\hline Trigo área (mill. tn) & 3.28 & 1.5 & 2.15 \\
\hline Soja producción (mill. tn) & 15.4 & 38.4 & 75.3 \\
\hline Sojárea (mill. tn) & 9.7 & 14 & 24.2 \\
\hline
\end{tabular}

Fuente: elaboración propia con base en datos del Censo Nacional Agropecuario 2006 (IBGE).

\section{Ganadería de carne y leche}

En cuanto a la evolución del sector ganadero vacuno en Argentina, tanto el stock como el consumo y las exportaciones registran un fuerte incremento después de la crisis de 2001 . El stock y las exportaciones empezaron a disminuir a partir de 2006/07, con una posterior caída drástica. El consumo se incrementó de 2006 a 2007 y experimentó una caída fuertísima a partir de 2009, de la que no ha logrado recuperarse. La situación de la ganadería argentina obedeció a la liquidación de stock producida y suba posterior de los precios de la carne, a raíz de las políticas públicas argentinas que privilegiaron el consumo interno sobre las exportaciones en 2006, sin que esa política evitara la reducción del consumo interno.

Así, mientras en Argentina se iba perdiendo en cabezas de ganado el equivalente al rodeo completo de Uruguay, y caían drásticamente el consumo interno y las exportaciones, en Brasil se mantuvo constante la tendencia al aumento del rodeo, que hoy supera los 200 millones 
Tabla 2.

Argentina: producción 1990-2010

\begin{tabular}{|c|c|c|c|}
\hline PRODUCTO & $\mathbf{1 9 9 0 / 1 9 9 1}$ & $\mathbf{2 0 0 0 / 2 0 0 1}$ & $\mathbf{2 0 1 0 / 2 0 1 0}$ \\
\hline Leche (mill. It) & 6.13 & 9.8 & 10.0 \\
\hline Carne vacuna (mill. tn) & 3.0 & 2.72 & 3.6 \\
\hline Stock vacuno (mill. cabezas) & 51.6 & 48.7 & 48.9 \\
\hline Máiz producción (mill. tn) & 7.7 & 15.3 & 23.7 \\
\hline Maízárea (mill. ha) & 2.2 & 3.5 & 4.6 \\
\hline Trigo producción (mill. tn) & 10.9 & 15.9 & 15.8 \\
\hline Trigo área (mill. tn) & 6.2 & 6.5 & 4.6 \\
\hline Soja producción (mill. tn) & 10.8 & 26.8 & 48.9 \\
\hline Soja área (mill. tn) & 5 & 10 & 18.9 \\
\hline
\end{tabular}

Fuente: elaboración propia con base en datos del Minagri.

de cabezas. En el caso de este país, la reaparición de la fiebre aftosa perjudicó seriamente su desempeño hasta 2003, cuando consiguió revertir esta situación, y, además, llegó a ser el principal exportador mundial de carne, desplazando a líderes indiscutidos hasta entonces, como Australia y Estados Unidos. En la actualidad, Brasil volvió a replegar esa posición, pero debido al gran crecimiento de su demanda interna por la suba del poder adquisitivo de la población, que le deja menores saldos para exportar. De todos modos, ya ronda los diez millones de toneladas de carne vacuna anuales de producción, y más de veinticinco millones, si se incluyen la carne de pollo y la de cerdo, versus una Argentina en retroceso que apenas roza los 2,5 millones de toneladas/año.

Como dato comparativo de fuerte impacto negativo para Argentina, ambos países contaban con un rodeo similar a mediados del siglo pasado, cuando, en los años cincuenta, rondaban los cincuenta y ocho/sesenta millones de cabezas. Además, Brasil empezó a exportar carne vacuna recién en 1914, mientras que para Argentina este producto fue el más emblemático y por el que se le reconoció desde inicios del siglo XX en el ámbito mundial.

En Brasil, desde 1990 la producción de carne ha logrado expandirse a una tasa constante de alrededor de $20 \%$ en cada una de las décadas, producto tanto del aumento casi permanente del rodeo como de la mejora en los índices de productividad, lo que le permitió afrontar el crecimiento de la demanda interna y el de las ventas al exterior. El hecho de que Brasil ya figuraba como un gran proveedor mundial de otras carnes, por la de pollo y la de cerdo, solo facilitó su irrupción en el mercado de la carne vacuna. Así lo expresó el director de Sustentabilidad de la Associação Brasileira das Indústrias Exportadoras de Carnes (Abiec), Fernando Sampaio, en Buenos Aires en 2010, citando un crecimiento del sector bovino en su país, 1975 a 2007, de un $227 \%$, mientras que el rebaño nacional de ganado casi se dobló superando los 200 millones de cabezas (Sampaio, 2010).

Pero no solo la carne vacuna logró esta expansión extraordinaria, con un gran esfuerzo competitivo en el país vecino; como se mencionó, también lo hicieron la producción y exportación de cerdos y de aves, que también crecieron agresivamente en los últimos quince años. Un hecho por destacar es que, de 2000 a 2008, la producción brasileña de cerdos aumentó el 
$19,5 \%$, pero el consumo interno solo lo hizo un 3,9\%, por lo que se debe concluir que la demanda externa fue sostenida por calidad de producto y bajo precio. En el ámbito internacional, Brasil se ha posicionado como el mayor exportador de carne de aves y vacuna, el cuarto exportador de carne de cerdo, y ocupa el 2,9\% de la producción mundial de porcinos. En esta posición de liderazgo alcanzada se reflejan todos los esfuerzos realizados por los productores brasileños y el Gobierno en las áreas de genética, nutrición, gestión y atención a los patrones mundiales de calidad.

Comparativamente, además, si bien Brasil cuenta con amplios programas de apoyo al sector, los productores debieron afrontar en el período considerado los altos precios de la energía - subsidiada en Argentina - y los persistentemente altos precios de los granos, insumo básico para la producción de leche, cerdos, pollos, y para completar el engorde de los vacunos, tema que en Argentina cuenta con la "ventaja" del subsidio implícito que representan las retenciones de $22 \%$ a $35 \%$ en cereales y oleaginosas. Como compensación, parcial, Brasil no aplica retenciones a sus exportaciones de carne ni de otros productos, lo que sí sucede en Argentina desde 2002, con porcentajes que van desde 5\% a 15\%, según el producto.

Un párrafo aparte merece la lechería, que, a pesar de no contar con un clima adecuado en Brasil, creció prácticamente el $100 \%$ en las dos décadas consideradas, llegando a los treinta mil millones anuales de litros de producción, lo que llevó a que ese país acotara sensiblemente sus tradicionales importaciones de este producto llegando, incluso, a exportar. Argentina, mientras tanto, creció solo $60 \%$, manteniéndose estancada en los volúmenes, casi desde fines de los años noventa, en unos diez mil millones de litros, mientras cayó sensiblemente la cantidad de establecimientos productivos que, en aquel momento, superaban holgadamente los veinte mil tambos, cifra que ahora se redujo a alrededor de diez mil.

Esta situación de disminución argentina fue determinada, entre otras cosas, porque tanto la producción de carne como la de leche son, comparativamente, más demandantes de mano de obra que la agricultura de cosecha, aspecto que fue muy castigado en los últimos años, comparativamente, con muy altos impuestos al trabajo.

En el caso argentino se dio, además, una mayor intervención oficial en los mercados, tanto internos como externos, que fijó precios de venta que determinaron fuertes transferencias de recursos a otros sectores, como el control de precios en góndola, el control de precios a los tambos, los precios "de corte" inferiores para exportar a los del mercado mundial, y otros, además de las retenciones a las exportaciones que también se aplican a este rubro.

\section{Argentina y Brasil agroindustriales veinte años después}

Un análisis retrospectivo muestra que Brasil ganó lugar como base productiva manufacturera en la región y buscó el autoabastecimiento en productos agrícolas, mientras que los países más pequeños, Uruguay y Paraguay, vieron acentuar el peso del sector agropecuario, en general, y el de la soja, en particular, llegando el sector a un 31,4\% del PBI paraguayo y 12,6\% del PBI uruguayo, mientras que tanto en Argentina como en Brasil es menor al 10\%.

Los avances en Argentina y Brasil de la producción de soja se basaron en semillas transgénicas, técnicas de siembra directa y uso de biocidas y fertilizantes, por un lado, y por otro lado, 
en nuevas formas de organización de la producción, con una mayor tendencia hacia la tercerización de actividades y, por supuesto, el alza sistemática de los precios internacionales por un corto período, en 1996/98,y luego, tras una abrupta caída en 2001/2, la recuperación casi continua desde 2004 en adelante. De todos modos, el sector agropecuario como tal tendió a ganar lugar en las estructuras productivas de los cuatro países. El alza de los precios internacionales motorizó fuertes inversiones de capital destinado a la producción agrícola para expandir las exportaciones extrazona. En este sentido, las IED tuvieron una etapa de incremento sostenido hasta 1998 en Argentina y Brasil. A partir de entonces, como hemos visto, fue Brasil quien atrajo un porcentaje muy alto de los capitales llegados a la región.

El desarrollo del sector agropecuario brasileño es actualmente muy destacado y es incentivado desde su Gobierno con fuertes ayudas. Así lo manifestó el agregado agrícola argentino en Brasil, Lic. Fernando Urbani, quien en un Informe de 2010 decía que:

El sector agropecuario brasileño muestra un alto grado de subsidio gubernamental. En la campaña 2009/2010, se destinarán 107,5 billones de Reales para apoyar la agricultura comercial y familiar. Este presupuesto es utilizado, principalmente, para garantizar precios mínimos y oferta de créditos a tasas subsidiados.

En ese contexto, se estima que Brasil producirá en la campaña 2009/2010 aproximadamente 143 millones de toneladas de granos en un área sembrada de 47,65 miIlones de hectáreas. En cuanto a ganadería, Brasil tiene el mayor stock del mundo, con un plantel de más 191 millones de cabezas de bovinos. (Urbani, 2010, p. 3)

Por su parte, la pérdida relativa de relevancia de Argentina respecto de Brasil durante los años ochenta se revirtió en la primera mitad de la década siguiente, debido básicamente a los resultados de la integración. Pero en la segunda parte de los noventa, la economía argentina se rezagó un tanto, y las diferencias se incrementaron. Si bien a partir de 2002 Argentina retomó el crecimiento de las exportaciones agroindustriales, buena parte del mismo se justificaría por la casi ininterrumpida suba de precios internacionales. En la actualidad, y con una economía brasileña que ha tomado una gran distancia de la argentina, que, además, no acompaña a su vecino, el proyecto de integración parece diluirse por falta de interés de los participantes, traducido en incumplimientos constantes de las reglas fijadas. Pareciera haberse perdido el objetivo del "regionalismo abierto".

Como conclusión, se podría decir que, en los últimos veinte años, se produjo una serie de avances técnicos que impulsaron una sustancial mejora de la productividad, especialmente, agrícola. En primer lugar, se registró la apertura económica de la región; luego, el avance tecnológico geométrico, que posibilitó mayores rindes e incorporación de nuevas tierras a la producción. Por el lado de la oferta, se implementaron nuevas tecnologías vinculadas, fundamentalmente, al uso de agroquímicos y semillas transgénicas, y al desarrollo de la siembra directa. Por el lado de la demanda, el crecimiento continuo de China e India tuvo como correlato un fuerte crecimiento de la demanda de alimentos. Se generó una tendencia a la mayor ingesta proteica con carnes, lácteos y aceites, lo que impulsó al alza los precios de las materias primas alimenticias en el ámbito internacional, lo que benefició en especial a países proveedores de alimentos, como los del Mercosur. 
Además, ante la caída de las tasas de interés y los programas de monetización de la Federal Reserve System (FED), muchos fondos de inversión se volcaron hacia las commodities como resguardo ante la incertidumbre y la volatilidad, elevando adicionalmente los precios y posibilitando un vuelco muy grande de capital hacia la agricultura.En este contexto, Argentina y Brasil se posicionaron como los países con mayores posibilidades de crecimiento al disponer de grandes extensiones de tierra cultivable.

La soja fue el cultivo "estrella" de los últimos años en la región, capitalizando todos estos avances estructurales. Argentina y Brasil se situaron a la cabeza de un proceso global, signado por condiciones favorables para la producción de commodities. Sin embargo, fue el sector sojero argentino el que tomó la delantera a mediados de los años noventa, con la aprobación del primer transgénico de la región, la soja RR, que permitió un desarrollo exponencial del cultivo. Se estima que la región alcanzará el 75\% de las exportaciones mundiales incidiendo, más aún, en la fijación de los precios del mercado mundial.

La producción en Argentina subió un 344\%, si se compara la actualidad con el promedio de los primeros cinco años de la década de 1990, pasando de once millones de toneladas a alrededor de cincuenta millones, con una expansión de la superficie sembrada de $276 \%$. La superficie aplicada al cultivo de soja pasó de aproximadamente 5 millones de hectáreas en 1990 a más de 18 millones en las últimas campañas. Esto representa algo más del 50\% del área cultivable argentina.

Brasil, por su parte, en igual período, incrementó un 349\% su producción, con una cosecha de 75 millones de toneladas en 2010/2011 y un avance de 127\% en la superficie dedicada al cultivo. En este país, la soja pasó a representar el $48,8 \%$ de la superficie total sembrada de granos.

A pesar de los volúmenes brasileños, Argentina sigue ocupando el primer lugar en la exportación de derivados industriales. En la última campaña, casi el 50\% de la harina de soja exportada mundialmente salió de los puertos argentinos; otro tanto le correspondió al aceite de la oleaginosa, que acaparó casi el 55\% del total comercializado.

\section{Perspectivas a mediano plazo}

Queda claro que el sector agroindustrial, tanto de Brasil como de Argentina, va a seguir creciendo. De hecho, el principal socio del Mercosur ya alcanzó el primer lugar como exportador agroalimentario mundial, y si la diferencia no es mayor aún, se debe solo al fuerte crecimiento que viene registrando de su propia demanda interna, lo que acota lógicamente sus márgenes exportables.

Argentina, por su parte, deberá encarar correcciones para no seguir registrando pérdidas respecto a su verdadero potencial productivo. Estas, a su vez, le están haciendo perder posiciones relativas respecto a su socio, Brasil.

A futuro, surgen algunos alertas. Un estudio encargado por el Ministerio de Ciencia y Tecnología de Argentina de 2008 sostiene que será imposible mantener la competitividad internacional y el perfil exportador agropecuario a futuro sin un fuerte desarrollo de la biotecnolo- 
gía (Regúnaga, Báez, Ganduglia y Massot, 2008, pp. 29-30).Además, en su política comercial es indispensable que Argentina diseñe estrategias de relaciones internacionales que posicionen al país dentro del contexto mundial. Por su parte, respecto a las debilidades, algunos sectores industriales como el de maquinaria agrícola, en 2010, destacaban que las mismas estaban en la falta de clima de negocios (previsibilidad, regulaciones, parámetros macroeconómicos, etcétera) y en la frágil articulación entre la esfera pública y la privada. Además, agregaban la dificultad de financiamiento y dotación de recursos humanos (Anlló, Bisang y Campi, 2010, pp. 217 y ss.).

Entre los obstáculos al crecimiento se pueden mencionar la escasa inversión, el inseguro abastecimiento de energía, las malas expectativas de las empresas, alta presión tributaria, escasez de crédito, dificultades en la infraestructura de caminos y transporte, un tipo de cambio menos competitivo y las restricciones con cupos a las exportaciones. Estas razones, entre otras, hacen que todavía no se haya podido articular una política agropecuaria común en el Mercosur, más allá de posiciones generales como denunciar los subsidios y el dumping agrícola de los países desarrollados.

En cualquier caso, es importante destacar que, con o sin políticas agropecuarias explícitas, en la región se ha dado un proceso que favoreció a las empresas de mayor escala, con una tendencia a la desaparición de los productores más pequeños.

Otro desafío común a ambos se centra en la intensificación en el uso de procesos productivos cada vez más "amigables" con el ambiente, que ya se ha convertido en un requerimiento creciente en los mercados internacionales, y en la mayor reposición de los nutrientes del suelo que se extraen en la producción. En este sentido, en Argentina, apenas se devuelve al suelo alrededor de una cuarta parte de los nutrientes que se extraen en cada cosecha, razón por la cual algunos sostienen que, en realidad, "se exporta fertilidad", o que "el suelo argentino se va por los puertos".

El país, a su vez, se encuentra ante el dilema de mantenerse en el camino centrado solo en la expansión económica, o tomar un rumbo basado en la multiplicación de riqueza sustentable que logre crear las condiciones para el progreso social. El dilema es: crecimiento o desarrollo. Hasta el momento, el Gobierno argentino privilegió el primer sendero: la expansión económica a tasas de entre un $8 \%$ y $9 \%$ en los últimos años ha postergado la definición de un proyecto de largo plazo. El desarrollo debe beneficiar a la mayoría de la población, y no limitarse a la modernización del capital o solo al beneficio de algún sector.

Brasil, por su parte, con ciento sesenta millones de toneladas de producción anual de granos (setenta de ellos de soja) en la última campaña, y más de veintinueve millones de toneladas de carnes (vacuna, aviar y de cerdo), entre otros rubros, se convirtió en las últimas dos décadas en el principal exportador mundial de alimentos, con ventas anuales por treinta y siete mil millones de dólares, desplazando incluso a Estados Unidos.

En el caso del principal socio del Mercosur, se ve una decisión estratégica muy clara, que es consolidar ahora esta posición. De hecho, las políticas adoptadas de fuerte respaldo a la producción le permitieron "compensar" desventajas competitivas frente a países en mejores condiciones agroecológicas como Argentina, para las producciones templadas. Dicho de otra 
124.

forma, mientras que Brasil buscó el crecimiento genuino, Argentina "desplazó" actividades al priorizar el corto plazo, con miras solo al mercado interno, lo que la llevó hasta el extremo del cierre de las exportaciones de su alimento más emblemático, la carne, en marzo de 2006.

Este comportamiento contrapuesto determinó que mientras Brasil "potenciaba" sus resultados y compensaba sus déficits productivos, Argentina, por el contrario, "frenaba" sus posibilidades, ya que, aun en los rubros que muestran crecimiento, como soja o maíz, los resultados deberían ser hoy muy superiores a los logrados, puesto que todavía no alcanzan para superar estructuralmente los cien millones de toneladas de granos anuales, cuando, con la tecnología disponible y las condiciones agroecológicas del país, la cosecha de granos debería ubicarse, por lo menos, en los ciento veinticinco/ciento treinta millones de toneladas por año.

Sin embargo, según algunos analistas económicos, la inestabilidad de las reglas de juego, que determinaron el retiro de Argentina de capitales brasileños que habían hecho inversiones frigoríficas; las retenciones, prohibiciones, la sobrecarga tributaria y la concentración de subsidios en zonas urbanas, especialmente del Gran Buenos Aires, versus Brasil, que los aplica a la producción, determinaron que el principal socio del Mercosur sacara una gran ventaja a Argentina en las últimas dos décadas, la que debería ser mucho menor, a la luz del potencial argentino, las mucho mejores condiciones agroecológicas para las producciones templadas, como trigo, leche, carne, etcétera, que determinan una mayor productividad, y el gran conocimiento en la materia de la población rural. Y esto, sin mencionar la extraordinaria coyuntura internacional de prácticamente la última década, con precios de alimentos inéditos, casi aprovechada a pleno por Brasil y desaprovechada por Argentina.

Así, mientras Brasil fortaleció la producción de sus productos tradicionales tropicales, e incorporó cantidad de otros nuevos como manzanas, vinos, leche, carne vacuna, y hasta trigo, entre otros -lo que le permitió convertirse en el principal exportador mundial de alimentos-, en Argentina sucedió exactamente lo contrario, con fuertes retrocesos en rubros tradicionales como la carne y el trigo. Simultáneamente, se trasladó buena parte de la inversión en estas actividades y de la ocupación territorial a la soja provocando transferencias y desfases riesgosos.

\section{Referencias}

Anllo, G., Bisang, R. y Campi, M. (2010). Cadenas de valor en la agroindustria. En B. Kosacoff y R. Mercado (eds.), Argentina ante la nueva industrialización de la producción. Crisis y oportunidades. Buenos Aires: Ed. Cepal/PNUD.

Cantamuto, A. (2005). Panorama 2005. El nuevo patrón de desarrollo en agricultura en América Latina y el Caribe.S antiago de Chile: Ed. Naciones Unidas.

Cisneros, A. y Piñeiro Iñíguez, C. (1994). Del ABC al Mercosur, La integración latinoamericana en la doctrina y praxis del peronismo. Buenos Aires: ISEN, Nuevo Hacer, Grupo Editor Latinoamericano. 
Constantino, A. y Cantamutto, F. (2010). El Mercosur agrario ¿integración para quién? Iconos: Revista de Ciencias Sociales, 38, pp: 67-80.

Evia, G. y Lapitz, R. (2004). Las contradicciones del éxito del Mercosur con la soja y la carne. Montevideo: Fundación Futuro Latinoamericano y Fundación Tinker.

Guadagni, A. (1992). Mercosur: una herramienta de desarrollo. En El Mercado Común del Sur. Buenos Aires: CEl. pp. 416-444.

Instituto Brasileiro de Geografía e Estadística -IBGE.

Lapitz, R.,Evia, G. y Gudynas, E. (2004). Soja y carne en el Mercosur, Comercio, ambiente y desarroIlo agropecuario. Editorial Coscoroba.

Machinandearena de Devoto, L. (2000). Historia del Mercosur: desde su fundación hasta el año 2000. Buenos Aires: Libros del CEIEG.

Ministerio de Agricultura, Ganadería y Pesca de la Argentina.

Peña, F., (1992). La Cumbre de Las Leñas y el futuro del Mercosur. En Felix Peña Artículos y Notas de Prensa. Buenos Aires. CEI. A2. № 28. pp. 73-80.

Peña, F. (2003). Momentos y perspectivas. La Argentina en el mundo y en América Latina. Buenos Aires: Universidad de Tres de Febrero (UNTREF).

Rapoport, M. (2005). Historia económica, política y social de la Argentina (1880-2003). Buenos Aires: Editorial Ariel.

Regúnaga, M. (coord.), Báez, G., Ganduglia, F. y Massot, M. A. (2008). Diagnóstico y estrategias para la mejora de la competitividad de la agricultura argentina. Buenos Aires: CARI, FAO, IICA.

Roppa, L. (2009). La producción de cerdos en Brasil; producción y consumo. Los Expertos Opinan.

Rusell, R. (1994). Los ejes estructurantes de la política exterior argentina: apuntes para un debate. Buenos Aires: Ed. Flacso.

Sampaio, F. (7/10/2010). Productores de ganado del Brasil aumentarán producción pese a limitaciones. Afecht.

Segrelles Serrano, J. A. (2003). Agricultura y territorio en el Mercosur. Revista Bibliográfica de Geografía y Ciencias Sociales, http://www.ub.edu/geocrit/b3w-483.htm

Sica, D. (2006). Mercosur: evolución y perspectivas, pp. 4-51. http://www.memorial.org.br/imagens_site/noticia/000590/Sica_Informe_Seminario_Memorial_Final.pdf

Sirlin, P. (2000). Impactos estáticos y dinámicos del proceso de apertura e integración de la década del '90: el caso de la industria molinera. Buenos Aires: Centro de Estudios de la Estructura Económica.

Urbani, F. (2010), Alimentos para el socio: el comercio agropecuario con Brasil. Revista Alimentos Argentinos. No 48. Buenos Aires: Ed. Minagri. pp. 65-67. 